\title{
A study of two Chinese patients with tetrasomy and pentasomy 15q11q13 including Prader-Willi/ Angelman syndrome critical region present with developmental delays and mental impairment
}

Jing Yang ${ }^{1 \dagger}$, Yongchen Yang ${ }^{2 \dagger}$, Yi Huang ${ }^{1}$, Yan Hu${ }^{3}, X_{i}$ Chen ${ }^{1}$, Hengjuan Sun ${ }^{1}$, Zhibao Lv², Qian Cheng ${ }^{3 *}$ and Liming Bao ${ }^{1,2,4^{*}}$

\begin{abstract}
Background: The proximal chromosome $15 q$ is prone to unequal crossover, leading to rearrangements. Although $15 q 11$ 113 duplications are common in patients with developmental delays and mental impairment, 15q aneusomies resulting in greater or equal to 4 copies of $15 q 11 q 13$ are rare and no pentasomy $15 q 11$ q13 has been reported in the literature. Thus far, all reported high copy number 15q11q13 cases are from the West populations and no such study in Chinese patients have been documented. Dosage-response pattern of high copy number 15q11q13 on clinical presentations is still a subject for further study.

Case Presentation: In this study, we characterized two Han Chinese patients with high copy number 15q11q13. Using chromosome banding, high resolution SNP-based cytogenomic array, Fluorescence in situ hybridization, and PCR-based microsatellite analysis, we identified two patients with tetrasomy $15 q 11 q 13$ and pentasomy $15 q 11$ q13. Both 15q11q13 aneusomies resulted from a maternally inherited supernumerary marker chromosome 15, and each was composed of two different sized 15q11q13 segments covering the Prader-Willi/Angelman critical region: one being about $10 \mathrm{Mb}$ with breakpoints at BP1 and BP5 regions on 15q11 and 15q13, respectively, and another about $8 \mathrm{Mb}$ in size with breakpoints at BP1 and BP4 regions on 15q. Both patients presented with similar clinical features that included neurodevelopmental delays, mental impairment, speech and autistic behavior, and mild dysmorphism. The patient with pentasomy 15q11q13 was more severely affected than the patient with tetrasomy 15q11q13. Low birth weight was noted in patient with pentasomy 15q1q13.

(Continued on next page)
\end{abstract}

\footnotetext{
* Correspondence: chqq5@126.com; Liming.Bao@cchmc.org

${ }^{\dagger}$ Equal contributors

${ }^{3}$ Department of Child Health Care and Growth Developmental and

Psychological Health Center, Children's Hospital of Chongqing Medical

University, Chongqing, China

${ }^{1}$ Center for Clinical Molecular Medicine; Ministry of Education Key Laboratory

of Child Development and Disorders; Key Laboratory of Pediatrics in

Chongqing; Chongqing International Science and Technology Cooperation

Center for Child Development and Disorders, Children's Hospital of

Chongqing Medical University, Chongqing, China

Full list of author information is available at the end of the article
} 
(Continued from previous page)

Conclusions: To the best of our knowledge, this is the first case of pentasomy 15q11q13 and the first study of high copy number 15q11q13 in Han Chinese patients. Our findings demonstrate that patients with tetrasomy and pentasomy of chromosome 15q11q13 share similar spectrum of phenotypes reported in other high copy number 15q11q13 patients in the West, and positive correlation between 15q11q13 copy number and degree of severity of clinical phenotypes. Low birth weight observed in the pentasomy 15q11q13 patient was not reported in other patients with high copy number $15 q 11$ 13. Additional studies would be necessary to further characterize high copy number 15q11q13 aneusomies.

Keywords: Chromosome 15, Cytogenomic array, Copy number, Pentasomy, Tetrasomy

\section{Background}

The proximal long arm of chromosome 15 (15q) contains repeat sequences that are prone to misalignments and unequal crossover during meiosis, leading to deletions, duplications, and supernumerary chromosomes [1-4]. Deletions of $15 q 11 q 13$ involving the Prader-Willi/Angelman syndrome critical region (PWACR) may cause Prader-Willi syndrome [MIM 176270] and Angelman syndrome [MIM 105830], depending on the parental origin [1]. Duplications of 15q11q13, particularly of maternal origin, are associated with clinical features, including psychomotor delays, cognitive disability, epilepsy, growth retardation, and behavioral problems $[3,5]$. Derivative chromosomes $15 \mathrm{q}$, mostly in a form of inverted duplication of $15 q$ (inv dup(15q)), are the most common supernumerary marker chromosomes (SMC) in humans [2,6,7]. Because of their clinical relevance, SMC15s spanning PWACR have been the focus of studies. Clinical features associated with tetrasomy $15 q 11 q 13$ have been reported but the underlying molecular characteristics have not been well characterized [2]. In contrast, aneusomies resulting in high copy number ( $\geq 4$ copies) $15 q 11 q 13$ segment, particularly greater than four copies, are rare. So far, only a dozen of hexasomy $15 \mathrm{q} 11 \mathrm{q} 13$ cases have been reported in the literature and none were pentasomy $15 q 11 q 13[4,8]$. All of the patients with high copy number $15 \mathrm{q} 11 \mathrm{q} 13$ reported thus far are from the West populations.

Most high copy number 15q11q13 aneusomies were studied by chromosome banding and fluorescence in situ hybridization (FISH), only few were analyzed at molecular levels. Recent advances in high density cytogenomic arrays provide powerful tools in detecting submicroscopic copy number change and delineating chromosome breakpoints. Here, we report a study of two Han Chinese patients with tetrasomy and pentasomy $15 \mathrm{q} 11 \mathrm{q} 13$ who present with developmental delays and cognitive disabilities.

\section{Case presentation}

\section{Clinical data}

Patients described in this study were enrolled in a large study of genomic aberrations in patients with developmental delays and intellectual disabilities at the Children's
Hospital of Chongqing Medical University, Chongqing, China. The study was approved by the hospital's Ethics Committee. Patient 1 was a 5 -year-old boy who had a history of developmental delays, intellectual disabilities, speech delay, and behavioral problems. He was the child of a healthy non-consanguineous couple. Family history was unremarkable. The gravida 1 para 1 mother was 25 , and the father, 28 , at the time of his birth. The mother had no history of miscarriages, and the pregnancy was uneventful. The patient was delivered at full-term, birth weight was $1,800 \mathrm{~g}$ ( $3^{\text {th }}$ percentile); length, $47 \mathrm{~cm}$ ( $25^{\text {th }}$ percentile); occipital frontal circumference, $32 \mathrm{~cm}$ (25 $5^{\text {th }}$ percentile). Resuscitation was performed at birth because of suffocation of unknown cause. No signs of asphyxia, jaundice, feeding problems, infections, or other problems were reported in the post-natal follow-up. Head control was achieved at 3 months; standing with aid at 1 year; and walking at 2 years. He started saying simple words at 2 years, and his speech was monosyllabic at 5 . Behavioral problems started at 2 . Evaluation at age of 5 showed that the patient was aggressive, short tempered, and had a tendency toward outbursts and being anger. He was hyperactive, impulsive, failed to follow instructions and rules, and was destructive. He had difficulties in focusing and was not able to finish tasks. He was easy to get into fights with peers, and did not do well in group activities. Physical exams showed slight microcephaly, thin upper lip, preauricular fistula, hypertelorism (Figures $1 \mathrm{~A}$ and $1 \mathrm{~B}$ ), moderate hypotonia at low extremities, and unable to stand on one foot and to run. Ligamentous laxity at the ankle joint was noted. At the age of 5, according to the scale of Gross Motor Function Measure [9], his scores for lying and rolling were 97; crawling and kneeling, 77; sitting, 100; standing, 79; and walking, 67. Based on Gesell Developmental Observation [10] (5 years old) his social adaptive skills were at 20 months; organizational skills, 15 months; motor skills, 18 months; and language skills, 19 months. Electroencephalography (EEG) study and cranial CT were normal, and no cardiac defects were detected. Patient 2 was born to a 34-year-old mother and 29-year-old father who are not related. The pregnancy and delivery were uneventful. His birth weight was $3,700 \mathrm{~g}\left(75^{\text {th }}\right.$ percentile $)$; length, $55 \mathrm{~cm}\left(50^{\text {th }}\right.$ 
percentile); head circumference, $35 \mathrm{~cm}\left(50^{\text {th }}\right.$ percentile). He did not walk until 2 years old, said simple words at 3 , and started to play with peers at 4 . At age of 8 years, he had difficulties in standing straight on one foot and had joint laxity. He showed hypertelorism, slightly anteverted nares, and low-set ears (Figures 1C and 1D). He had pathic facial expression and said simple sentences. By age of 9 years, his body weigh was $31.5 \mathrm{~kg}$ (95 ${ }^{\text {th }}$ percentile); height, $125 \mathrm{~cm}$ $\left(50^{\text {th }}\right.$ percentile); and head circumference, $53 \mathrm{~cm}\left(75^{\text {th }}\right.$ percentile). He had no problems in walking and running. He spoke simple sentences and was not able to repeat stories. The behavioral problems started at age of 2 years. He did not like to join group activities, and had tendency to be aggressive and destructive. Evaluations with the Weshsler Intelligence Scale for Children, fourth edition (WISC-IV) [11] showed his four index cores scores at age of 9 were: verbal comprehension, 45; perceptual reasoning, 40; working memory, 50; processing speed, 46 and full scale IQ, 41. EEG study and cranial CT were all normal.

\section{Genetic analysis}

Banded chromosome analysis of peripheral blood revealed each patient had a SMC15: a rea(15)(q11) or 47,XY,+rea (15)(q11) in patient 1 and an inv dup(15)(q11q13) or 47,XY, +inv dup(15)(q11q13) in patient 2 (Figure 2). Parental cytogenetic studies were normal. Genomic DNA from the patients was prepared and hybridized to SNP-based Cytoscan HD array (Affymetrix, USA). The cytogenomic arrays had 2.69 million markers covering the entire human genome with the average spatial distance between intragenic neighboring markers of 880 bases. Cel intensity or CEL files were created using Affymetrix GeneChip Command Console operating software and Chromosome Analysis Suite, according to manufacturer's protocols. Copy number changes were detected using a Hidden Markov Model algorithm [12]. Gene annotations were determined using build GRCh37/hg19. The array analysis revealed increases in 15q11q13 copy number in both patients. Patient 1 had 5 copies of 15q11.2q13.2 (7.72 Mb, chr15:22,619,671$30,342,457)$, and 3 copies of $15 \mathrm{q} 13.2 \mathrm{q} 13.3$ (2.89 Mb, chr15:30,342,458-32,411,629), indicating the SMC15 was comprised of 2 copies of $15 \mathrm{q} 11.2 \mathrm{q} 13.2$ (7.72 Mb, chr15:22,619,671-30,342,457) and 1 copy of $15 \mathrm{q} 11.2 \mathrm{q} 13.3$ (10.61 Mb, chr15:22,619,671-32,411,629) (Figure 3). Patient 2 had 4 copies of 15q11.2q13.1 (8.00 Mb, chr15:22,054,840$30,058,502)$ and 3 copies of $15 \mathrm{q} 13.1 \mathrm{q} 13.2$ (2.36 Mb, chr15:30,058,503-32,418,417), demonstrating that the inv dup(15q) contained one 8.00-Mb 15q11.2q13.1 segment (chr15:22,054,840-30,058,502) and another 10.36-Mb 15q11.2q13.3 segment (chr15:22,054,840-32,418,417) (Figure 4). FISH using the SNRPN probe (Abbott Molecular, USA) showed that in addition to 2 signals on normal chromosome 15 homologues, the SMC15 from patient 1 had 3 signals (Figures $2 b$ and $2 c$ ), and the inv dup(15q) from patient 2 had 2 signals (Figures $2 \mathrm{e}$ and $2 \mathrm{f})$, confirming the array findings of pentasomy and tetrasomy 15q11q13 in patients 1 and 2, respectively.

Parental origin of 15q11q13 aneusomies was analyzed by PCR-based genotyping of 12 STS loci on 15q11q13. Informative markers in patient $1, D 15 S 975, D 15 S 156 R$, D15S1043 and D15S123, showed 1 and 2 copies of paternal and maternal alleles, respectively, consistent with maternal inheritance of the SMC15. Informative alleles in patient 2, D15S817, D15S97 and D15S1048, were 1 and 2 copies of paternal and maternal alleles, respectively, also suggesting maternal origin of the inv dup(15) (Table 1). Quantitative PCR methylation analysis of SNRPN was performed to determine methylation pattern of 15q11q13. Patient genomic DNA was pre-treated with bisulfite using EZ DNA Methylation-Gold kit (Zymo, USA). Primers for methylated and unmethylated SNRPN

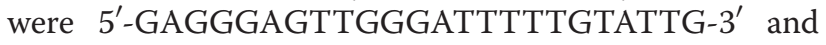
$5^{\prime}$-CCCAAACTATCTCTTAAAAAAAACCAC-3', and $5^{\prime}$-CTCCAAAACAAAAAACTTTA AAACCCAAATT CC- $3^{\prime}$ and $5^{\prime}$-GTGAGTTTGGTGTAGAGTGGAGT GGTTGTTG- $3^{\prime}$, respectively. The PCR conditions were 1 cycle at $95^{\circ} \mathrm{C}$ for $2 \mathrm{~min}$, and 45 cycles at $95^{\circ} \mathrm{C}$ for $15 \mathrm{~s}, 61^{\circ} \mathrm{C}$ for $30 \mathrm{~s}$, and $72^{\circ} \mathrm{C}$ for $45 \mathrm{~s}$. Each reaction was done in triplicate. The $\beta$-actin gene was used as an internal control. Both patients showed 1 copy of unmethylated (paternal pattern) $S N R P N$, and 4 and 3

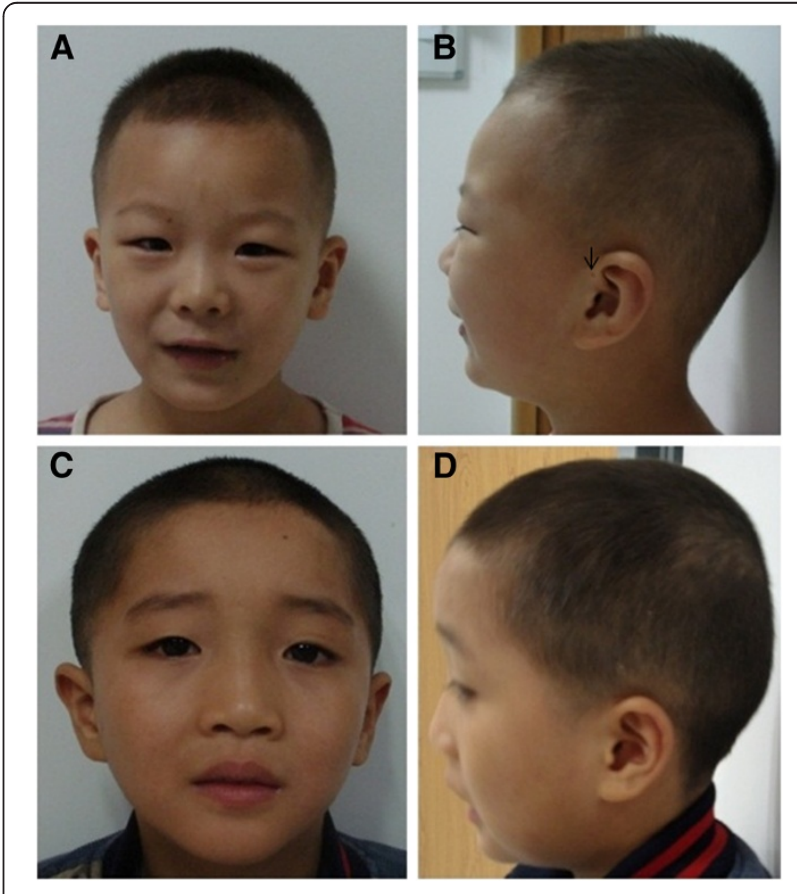

Figure 1 Patients photographs. Frontal $(\mathbf{A})$ and side $(\mathbf{B})$ views of patient 1 showing upper thin lip, preauricular fistula (arrowed) and hypertelorism. Frontal (C) and side (D) views of patient 2 showing hypertelorism, slightly anteverted nares and low-set ears. 
copies of methylated (maternal pattern) SNRPN in patients 1 and 2, respectively (data not shown), demonstrating maternal imprinting pattern of both SMC15s.

\section{Discussion}

Here, we report tetrasomy and pentasomy 15q11q13 in two Han Chinese patients. To the best of our knowledge, pentasomy 15q11q13 has not been reported in the literature and the work presents the results of the first study of high copy number 15q11q13 in Chinese patients. Most clinical features in our patients including neurodevelopmental delays, intellectual disabilities, speech problems, and mild dysmorphisms overlap with the ones previously described in high copy number $15 \mathrm{q} 11 \mathrm{q} 13$ patients in the West $[2,4,8,13]$. However, some differences were noted. Seizures are present in two-thirds of tetrasomy patients with the age of onset ranging from 6 months to 9 years $[2,8]$, and were also reported in ten patients with hexasomy 15q11q13 starting at less than 3 years old. Seizures had not been developed in our patients with tetrasomy and pentasomy 15q11q13 at age of 5 and 9 years, respectively. Vision deficits are uncommon in patients with trisomy and tetrasomy $15 \mathrm{q} 11 \mathrm{q} 13$, and were seen in 3 of 10 hexasomy patients $[2,8,14]$. Neither of the patients in this study had vision deficits. Severe developmental delays and intellectual disabilities are prominent features in hexasomy $15 \mathrm{q} 11 \mathrm{q} 13$, and the pentasomy patient reported here also showed profound developmental delays and cognitive deficits. Of 8 hexasomy patients whose language development was evaluated, 6 had absent or nonverbal speech and 2 was poor speech $[4,8]$. The pentasomy $15 \mathrm{q} 11 \mathrm{q} 13$ patient had severe speech problems and was only able to say simple words at 5 . The tetrasomy patient said simple sentences at 9. Although both patients reported in this study demonstrated behavioral problems overlapping with some features in patients with autism, the severity of psychomotor retardation in the patients was incompatible with a diagnosis of autism. Another notable feature in patient with pentasomy $15 \mathrm{q} 11 \mathrm{q} 13$ is low birth weight which was not reported in other patients with high copy number 15q11q13 $[8,15,16]$. Low birth weight might contribute to development delays. Clinical presentations observed in the patient with tetrasomy 15q11q13 overlap those described in Western patients with similar 15q aneusomy [2]. Although both tetrasomy and pentasomy patients shared similar clinical profiles, pentasomy patient appeared to be more severely affected than tetrasomy patient with regard to development delays and speech problems. These observations suggest that the increase in copy number in 15q11q13 aneusomies may not change clinical features, but result in increased severity. This is in agreement with previous reports from the studies of patients with no more than four copies of $15 q 11 q 13[2,4,8]$.

Clinical heterogeneity among patients with various $15 q 11$ q13 aneusomies may be attributed to differences in copy number, the extent of affected regions, parental origin, age at evaluation, available clinical information, and the presence of mosaicism. Study of additional cases with high copy number 15q11q13 would be necessary

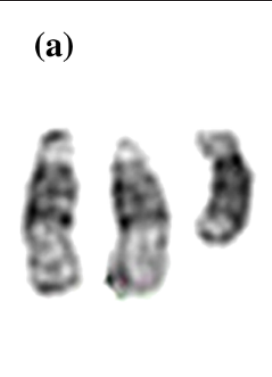

(d)

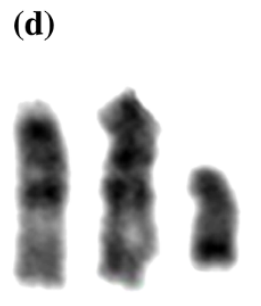

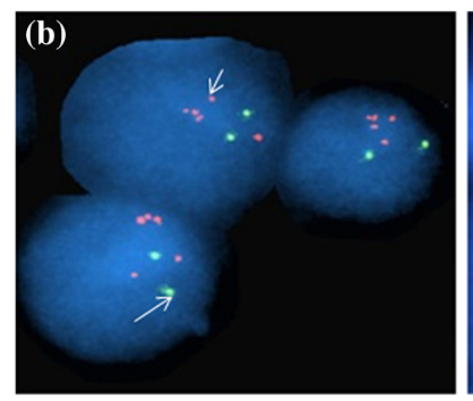
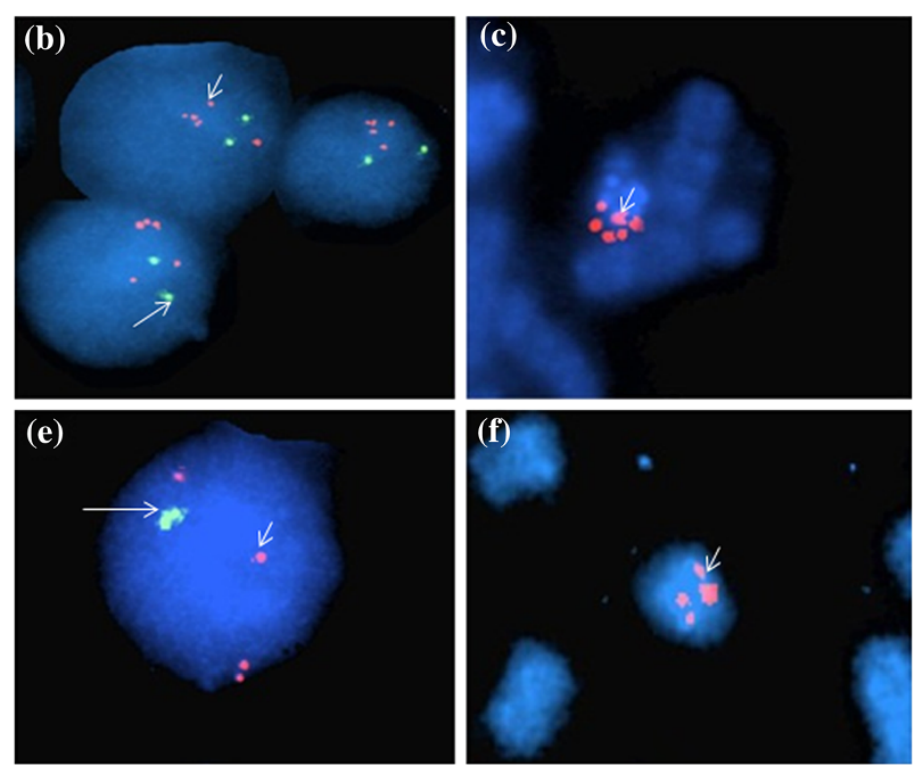

Figure 2 Partial karyotype and FISH analysis. Patient 1: (a) partial karyotype of derivative chromosome 15. FISH study using probes for SNRPN locus (orange, short arrows) at 15q11q13 and PML locus (green, long arrows) at 15q22: (b) interphase cells and (c) SMC15. Patient 2: (d) partial karyotype of inv dup(15), FISH using SNRPN and PML probes: (e) interphase cells and (f) inv dup(15q). 


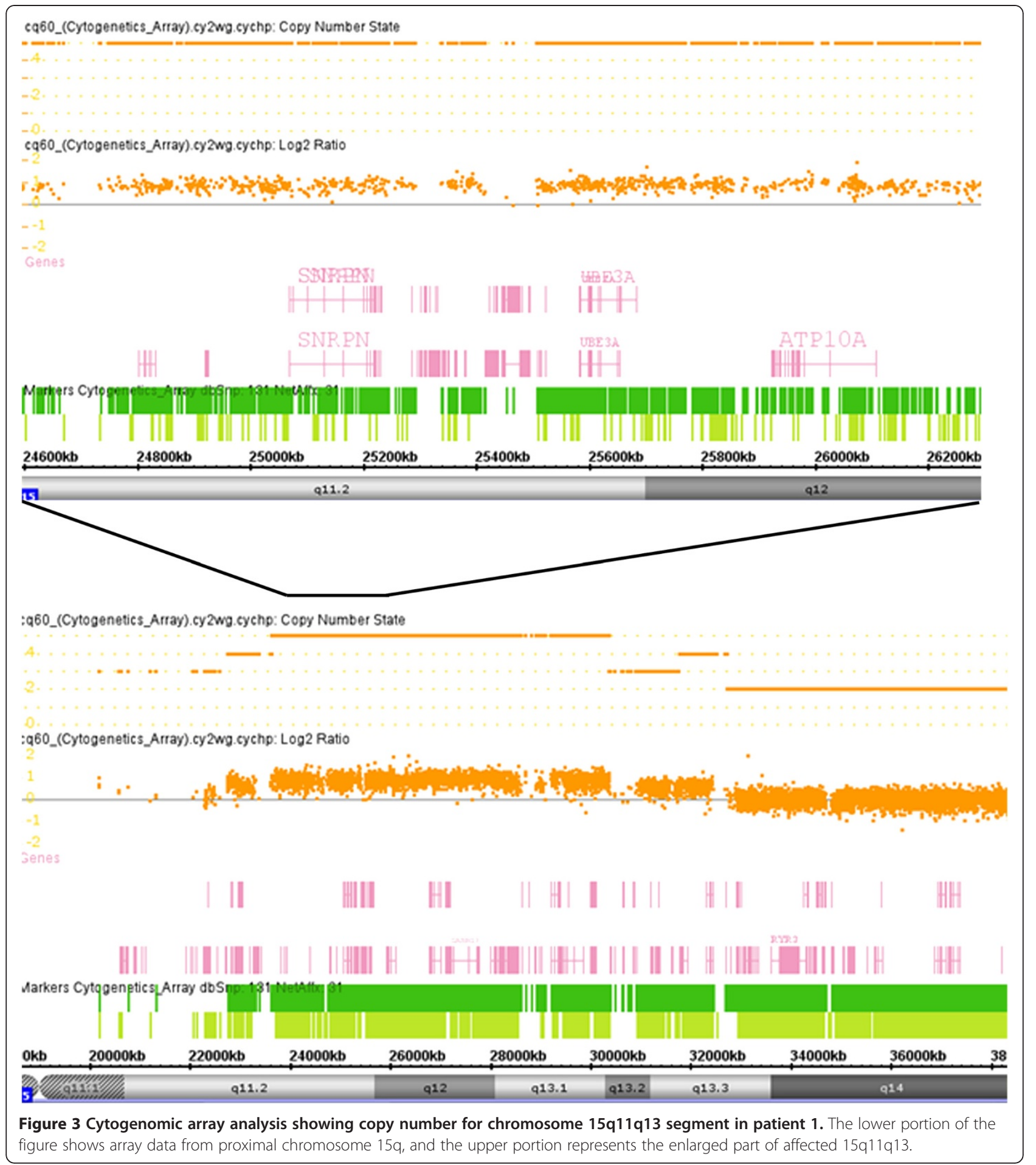

for a better understanding of clinical phenotypes associated with high copy number 15q11q13.

The pentasomy and tetrasomy 15q11q13 reported here were all materially inherited. Genes in the BP1-BP4 region that are maternally imprinted and paternally expressed, including MKRN3, NDN, MAGEL2 and $S N U R F / S N R P N$, are unlikely to contribute to the clinical phenotypes. However, genes that are maternally preferentially expressed in the brain, such as $U B E 3 A$ and $A T P 10 A$, may increase in expression and have clinical effects. Other genes in the region, including those for $\mathrm{GABA}_{\mathrm{A}}$ receptor subunits (GABRB3, GABRA5, and $G A B R G 3)$ and $A B P A 2$ are not subject to imprinting and are biallelically expressed. The $\mathrm{GABA}_{\mathrm{A}}$ receptor genes 


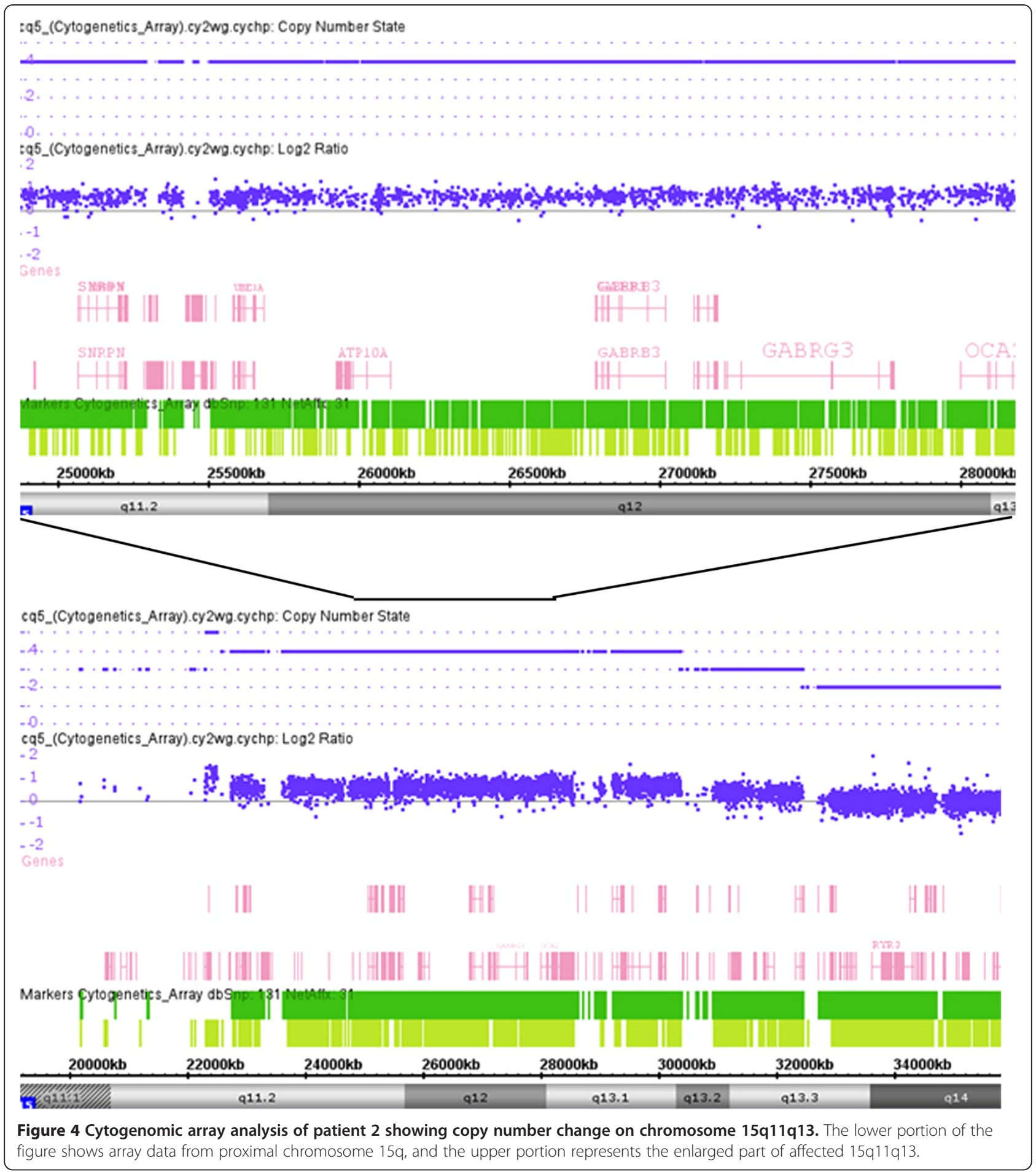

have been implicated in autism, and ABPA2 plays a critical role in synaptic vesicle exocytosis in the brain $[17,18]$. The CHRFAM7A and CHRNA7 genes that reside in the BP4-BP5 region have been linked to psychiatric disorders [19].

Most high copy number 15q11q13 abnormalities were studied by chromosome banding and/or FISH analysis, and few were characterized at molecular levels. Using high-resolution cytogenomic array, we were able to delineate high copy number 15q11q13 aneusomies at high precision (average spatial distance between intragenic neighboring markers: 880 bases). The breakpoints for the large 15q11q13 segments in both SMC15s fall into the same BP1 and BP5 regions on proximal 15q, while 
Table 1 Parental origin analysis of derivative chromosomes 15

\begin{tabular}{|c|c|c|c|c|c|c|}
\hline \multirow[b]{2}{*}{ STS } & \multicolumn{3}{|c|}{ Patient 1} & \multicolumn{3}{|c|}{ Patient 2} \\
\hline & Father & Mother & Proband & Father & Mother & Proband \\
\hline D15S541 & 1,2 & 1,2 & 1,2 & 1,1 & 1,2 & 1,2 \\
\hline D15S817 & 1,1 & 2,2 & 2,2 & 1,1 & 2,3 & $1,2,3$ \\
\hline GABR3 & 1,1 & 1,1 & 1,1 & 1,1 & 1,1 & 1,1 \\
\hline D15S97 & 1,2 & 1,2 & 1,2 & 1,2 & 3,4 & $1,3,4$ \\
\hline D15S975 & 1,2 & 3,4 & $1,3,4$ & 1,2 & 1,2 & 1,2 \\
\hline D15S156R & 1,2 & 3,4 & $1,3,4$ & 1,2 & 1,3 & 1,3 \\
\hline D15S815 & 1,1 & 1,1 & 1,1 & 1,2 & 1,3 & 1,3 \\
\hline D15S1048 & 1,2 & 1,2 & 1,2 & 1,1 & 2,3 & $1,2,3$ \\
\hline D15S1043 & 1,1 & 2,3 & $1,2,3$ & 1,2 & 1,3 & 1,3 \\
\hline D15S1013 & 1,2 & 1,1 & 1,2 & 1,1 & 2,2 & 1,2 \\
\hline D15S123 & 1,1 & 2,3 & $1,2,3$ & 1,2 & 1,2 & 1,2 \\
\hline D15S1007 & 1,2 & 1,2 & 1,1 & 1,2 & 1,2 & 1,2 \\
\hline
\end{tabular}

the breakpoints for small 15q11q13 segments were at $\mathrm{BP} 1$ and BP4 regions. The PWACR region $(4 \mathrm{Mb})$ is located between BP2 and BP3. Our results concur with the findings from previous studies that distal breakpoints for large 15q11q13 rearrangements often fall within BP4 or $\mathrm{BP} 5$ region and $\mathrm{BP} 4$ is more prone than BP5 to recombination [20-22].

\section{Conclusions}

We identified two Han Chinese patients with tetrasomy and pentasomy 15q11q13 containing PWACR. To the best of our knowledge, this is the first pentasomy 15q11q13 case and the first study of high copy number 15q11q13 in Chinese populations. Both 15q11q13 aneusomies were maternally inherited and composed of two sized 15q11q13 segments with similar proximal breakpoints at BP1 and distal breakpoints at BP4/BP5. The patients shared similar spectrum of clinical features including neurodevelopmental delays, intellectual disabilities, speech delay, autistic behaviors, and mild dysmorphism, with pentasomy patient being more severely affected than tetrasomy patient. Low birth weight observed in the pentasomy patient has not been previously described in patients with high copy number $15 \mathrm{q} 11 \mathrm{q} 13$. Our findings suggest positive relationship between 15q11q13 copy number and degree of severity of clinical presentations.

\section{Consent}

Written informed consents were obtained from patient parents for publication and the accompanying images. Copies of the written consents are available for review by the Editor-in-Chief of this journal.

\section{Abbreviations}

PWACR: Prader-Willi/Angelman syndrome critical region;

SMC: Supernumerary marker chromosome; PCR: Polymerase chain reaction; BP: Breakpoint.

\section{Competing interests}

The authors declaim no competing interests.

\section{Authors' contributions}

LB, QC and YH wrote the manuscript; LB, QC, YY, YH, and ZL participated in study design, data interpretation; JY, YH QC, XC, YH and HS collected data; $J Y, Y H, Y H, X C, Y Y$ and $H S$ conducted experiments and data analysis. All authors read and approved the final manuscript.

\section{Acknowledgments}

The authors are grateful to the patients and families for participating in the study. The study was supported in part by the grants from the Chongqing Science and Technology Commission (CSTC2009AB5217) and Shanghai Shen Kang Hospital Development Center (SHDC12007201).

\section{Author details}

${ }^{1}$ Center for Clinical Molecular Medicine; Ministry of Education Key Laboratory of Child Development and Disorders; Key Laboratory of Pediatrics in Chongqing; Chongqing International Science and Technology Cooperation Center for Child Development and Disorders, Children's Hospital of Chongqing Medical University, Chongqing, China. ${ }^{2}$ Shanghai Children's Hospital, Shanghai Children's Hospital Affiliated to Shanghai Jiao Tong University School of Medicine, Shanghai, China. ${ }^{3}$ Department of Child Health Care and Growth Developmental and Psychological Health Center, Children's Hospital of Chongqing Medical University, Chongqing, China. ${ }^{4}$ Division of Human Genetics, Department of Pediatrics, Cincinnati Children's Hospital Medical Center and University of Cincinnati College of Medicine, Cincinnati, $\mathrm{OH}$, USA.

Received: 14 September 2012 Accepted: 7 January 2013

Published: 15 January 2013

\section{References}

1. Knoll JH, Nicholls RD, Magenis RE, Graham JM Jr, Lalande M, Latt SA: Angelman and Prader-Willi syndromes share a common chromosome 15 deletion but differ in parental origin of the deletion. Am J Med Genet 1989, 32(2):285-290.

2. Battaglia A: The inv dup(15) or idic(15) syndrome: a clinically recognisable neurogenetic disorder. Brain Dev 2005, 27(5):365-369.

3. Ungaro P, Christian SL, Fantes JA, Mutirangura A, Black S, Reynolds J, Malcolm S, Dobyns WB, Ledbetter DH: Molecular characterisation of four cases of intrachromosomal triplication of chromosome 15q11-q14. J Med Genet 2001, 38(1):26-34.

4. Kraoua L, Chaabouni M, Ewers E, Chelly I, Ouertani I, Ben Jemaa L, Maazoul $\mathrm{F}$, Liehr T, Chaabouni H: Hexasomy of the Prader-Willi/Angelman critical region, including the OCA2 gene, in a patient with pigmentary dysplasia: case report. Eur J Med Genet 2011, 54(4):e446-450.

5. Crolla JA, Harvey JF, Sitch FL, Dennis NR: Supernumerary marker 15 chromosomes: a clinical, molecular and FISH approach to diagnosis and prognosis. Hum Genet 1995, 95(2):161-170.

6. Friedrich $U$, Nielsen $\mathrm{J}$ : Bisatellited extra small metacentric chromosome in newborns. Clin Genet 1974, 6(1):23-31.

7. Webb T: Inv dup(15) supernumerary marker chromosomes. J Med Genet 1994, 31(8):585-594.

8. Mann SM, Wang NJ, Liu DH, Wang L, Schultz RA, Dorrani N, Sigman M, Schanen NC: Supernumerary tricentric derivative chromosome 15 in two boys with intractable epilepsy: another mechanism for partial hexasomy. Hum Genet 2004, 115(2):104-111.

9. Russell DJ, Avery LM, Rosenbaum PL, Raina PS, Walter SD, Palisano RJ: Improved scaling of the gross motor function measure for children with cerebral palsy: evidence of reliability and validity. Phys Ther 2000, 80(9):873-885.

10. Gesell A: The clinical supervision of child development. Wis Med J 1949, 48(2):119-123.

11. Wechsler D: Wechesler Interlligence Scale for Children-Fourth Edition. San Antonin, TX: The Psychological Corporation; 2003. 
12. Arribas-Gil A, Matias C: A context dependent pair hidden Markov model for statistical alignment. Stat Appl Genet Mol Biol 2012, 11(1). Article 5.

13. Dennis NR, Veltman MW, Thompson R, Craig E, Bolton PF, Thomas NS: Clinical findings in 33 subjects with large supernumerary marker(15) chromosomes and 3 subjects with triplication of 15q11-q13. Am J Med Genet A 2006, 140(5):434-441.

14. Kitsiou-Tzeli S, Tzetis M, Sofocleous C, Vrettou C, Xaidara A, Giannikou K, Pampanos A, Mavrou A, Kanavakis E: De novo interstitial duplication of the 15q11.2-q14 PWS/AS region of maternal origin: Clinical description, array CGH analysis, and review of the literature. Am J Med Genet A 2010, 152A(8):1925-1932.

15. $L i \mathrm{H}, \mathrm{Ji} \mathrm{CY}$, Zong XN, Zhang YQ: Height and weight standardized growth charts for Chinese children and adolescents aged 0 to 18 years. Chin J Pediatr 2009, 47(7):487-492.

16. Li H: Growth standardized values and curves based on weight, length/ height and head circumference for Chinese children under 7 years of age. Chin J Pediatr 2009, 47(3):173-178.

17. Kim SA, Kim JH, Park M, Cho IH, Yoo HJ: Association of GABRB3 polymorphisms with autism spectrum disorders in Korean trios. Neuropsychobiology 2006, 54(3):160-165.

18. Sutcliffe JS, Han MK, Amin T, Kesterson RA, Nurmi EL: Partial duplication of the APBA2 gene in chromosome 15q13 corresponds to duplicon structures. BMC Genomics 2003, 4(1):15.

19. Olincy A, Harris JG, Johnson LL, Pender V, Kongs S, Allensworth D, Ellis J, Zerbe GO, Leonard S, Stevens KE, et al: Proof-of-concept trial of an alpha7 nicotinic agonist in schizophrenia. Arch Gen Psychiatry 2006, 63(6):630-638.

20. Pujana MA, Nadal M, Guitart M, Armengol L, Gratacos M, Estivill X: Human chromosome 15q11-q14 regions of rearrangements contain clusters of LCR15 duplicons. Eur J Hum Genet 2002, 10(1):26-35.

21. Mignon-Ravix C, Depetris D, Luciani JJ, Cuoco C, Krajewska-Walasek M, Missirian C, Collignon P, Delobel B, Croquette MF, Moncla A, et al: Recurrent rearrangements in the proximal 15q11-q14 region: a new breakpoint cluster specific to unbalanced translocations. Eur J Hum Genet 2007, 15(4):432-440.

22. Knoll JH, Nicholls RD, Magenis RE, Glatt K, Graham JM Jr, Kaplan L, Lalande $\mathrm{M}$ : Angelman syndrome: three molecular classes identified with chromosome 15q11q13-specific DNA markers. Am J Hum Genet 1990, 47(1):149-154.

doi:10.1186/1471-2350-14-9

Cite this article as: Yang et al:: A study of two Chinese patients with tetrasomy and pentasomy 15q11q13 including Prader-Willi/Angelman syndrome critical region present with developmental delays and mental impairment. BMC Medical Genetics 2013 14:9.

\section{Submit your next manuscript to BioMed Central and take full advantage of:}

- Convenient online submission

- Thorough peer review

- No space constraints or color figure charges

- Immediate publication on acceptance

- Inclusion in PubMed, CAS, Scopus and Google Scholar

- Research which is freely available for redistribution 